\title{
Target prediction in forward scattering radar
}

\begin{abstract}
This paper describes aspects of ground target detection using a Forward Scattering Radar (FSR). The problem of extracting the Doppler signature in different interference environments is addressed. Hilbert Transform and Wavelet have been used to predict the existence of target. The paper begins with a brief description of the system, followed by a more detailed analytical study of predicting the presence of target in FSR. A practical experimentation has been realised to evaluate the proposed algorithm.
\end{abstract}

Keyword: Forward scattering radar; Hilbert transform; Wavelet denoise 